BASIC RESEARCH

\title{
Changes in myocardial blood volume over a wide range of coronary driving pressures: role of capillaries beyond the autoregulatory range
}

\author{
D E Le, A R Jayaweera, K Wei, M P Coggins, J R Lindner, S Kaul
}

Heart 2004;90:1199-1205. doi: 10.1136/hrt.2003.020875

See end of article for authors' affiliations

Correspondence to:

Dr Sanjiv Kaul, University

of Virginia Health System,

Cardiovascular Division,

Box 800158 ,

Charlottesville, VA 22908-

0158, USA; sk@hscmail.

mcc.virginia.edu

Accepted

12 December 2003
Objective: To determine whether, when the vasomotor capacity of the coronary arterioles is exhausted at rest, myocardial blood volume decreases in order to maintain a normal capillary hydrostatic pressure, even at the expense of myocardial oxygen delivery.

Methods: 18 dogs were studied. In group $1(\mathrm{n}=9)$, coronary driving pressure (CDP) was reduced by 10$80 \mathrm{~mm} \mathrm{Hg}$ below normal by a stenosis; in group 2 ( $\mathrm{n}=9$ ), it was increased $20-80 \mathrm{~mm} \mathrm{Hg}$ above baseline by increasing aortic pressure with phenylephrine. Myocardial contrast echocardiography (MCE) was undertaken to measure the myocardial blood volume fraction and myocardial blood flow (MBF). Results: In group 1 dogs, as CDP was reduced, both coronary blood flow (CBF) and MBF decreased. Myocardial blood volume fraction also decreased and myocardial vascular resistance increased, while coronary sinus $\mathrm{PO}_{2}$ decreased. In group 2 dogs, as $\mathrm{CDP}$ was increased, epicardial CBF increased but MBF remained unchanged because of a decrease in myocardial blood volume fraction. Myocardial vascular resistance decreased, however, implying the presence of coronary arteriovenous shunting, which was supported by a progressive increase in the coronary sinus $\mathrm{PO}_{2}$.

Conclusions: When arteriolar tone is exhausted so that CBF becomes dependent on CDP, myocardial blood volume decreases in order to maintain a constant capillary hydrostatic pressure, which takes precedence over myocardial oxygen delivery. These novel findings implicate capillaries in the regulation of $\mathrm{CBF}$ beyond the autoregulatory range.

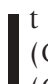
has been shown previously that coronary blood flow $(\mathrm{CBF})$ remains constant when coronary driving pressure (CDP) is varied between approximately $45-120 \mathrm{~mm}$ without changing myocardial work. ${ }^{1}$ This process of autoregulation is thought to be dependent primarily on small resistance

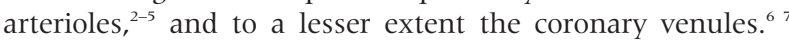
When CDP is altered to levels either below or above this autoregulatory range, $\mathrm{CBF}$ becomes pressure dependent.

An important role of autoregulation is the maintenance of capillary hydrostatic pressure (capHP) and homeostasis, which are essential for cell survival. It has been shown in the skeletal muscle that despite wide variations in perfusion pressure, the net transcapillary fluid movement remains remarkably stable, which implies that capHP stays relatively constant. ${ }^{8}$ The net transcapillary fluid movement remains stable even when perfusion pressure is varied beyond the lower and upper limits of the autoregulatory range. ${ }^{9}$ The only way capHP can remain constant when perfusion pressure either decreases below or increases above the autoregulatory range is by changes in capillary resistance.

We hypothesised that when the vasomotor capacity of the coronary arterioles is exhausted at rest (CDP below 45 or above $120 \mathrm{~mm} \mathrm{Hg}$ ) - that is, the CDP is beyond the autoregulatory range-capHP is maintained by changes in capillary blood volume (capBV) even at the expense of myocardial oxygen delivery. In order to test this hypothesis, we altered the CDP over a wide range and measured capBV using myocardial contrast echocardiography (MCE).

During MCE, gas filled microbubbles are injected intravenously and an ultrasound examination of the heart is carried out. $^{10}$ The degree of myocardial opacification during MCE is proportional to the concentration of microbubbles within the myocardium and represents relative myocardial blood volume (MBV). ${ }^{11}$ We have previously shown that changes in blood volume fraction measured using this technique correlate well with an independent method that measures capillary surface area. ${ }^{12}$ Although historically the term MBV has referred to blood residing within the entire coronary circulation, ${ }^{11}$ we now use it to denote blood present within the myocardial microvasculature itself, which includes arterioles, capillaries, and venules. ${ }^{8}$ We have assigned the term "coronary blood volume" to the blood present in the entire coronary circulation. ${ }^{11}$ Within the myocardium itself, $90 \%$ of the blood resides in capillaries $\left(\sim 2500-3000\right.$ per $\mathrm{mm}^{3}$ of tissue). ${ }^{8}$ Therefore MBV essentially represents capBV. Because the length of a single capillary does not change, a change in capillary volume will reflect a change in total capillary resistance. ${ }^{13}$ Thus changes in capillary resistance can be measured with MCE. ${ }^{14}$ We have also validated MCE as an accurate method for assessing myocardial blood flow $(\mathrm{MBF}){ }^{15}$

\section{METHODS}

\section{Animal preparation}

The study protocol was approved by the animal research committee at the University of Virginia and conformed to the American Heart Association Guidelines for the use of animals in research. We used 18 adult open chest anaesthetised dogs

Abbreviations: capBV, capillary blood volume; capHP, capillary hydrostatic pressure; $C B F$, coronary blood flow; $C D P$, coronary driving pressure; CVR, coronary vascular resistance; $M B F$, myocardial blood flow; MBV, myocardial blood volume; MCE, myocardial contrast echocardiography; $\mathrm{MVO}_{2}$, myocardial oxygen consumption; $\mathrm{MVR}$, myocardial vascular resistance. NOTE: "capillary" refers to only capillaries within the myocardium, "coronary" refers to epicardial coronary arteries, and "myocardial" refers to the entire microvasculature within the myocardium, which includes arterioles, capillaries, and venules. 
(30-35 kg) for the study, divided into two groups of nine dogs each. CDP was decreased below normal in group 1 and increased above normal in group 2. Catheters (7 French) were placed in both the femoral veins for microbubble infusion and administration of fluids and drugs as needed, and in the aortic root and the left atrium to measure pressures.

A left lateral thoracotomy was done in the fourth intercostal space and the heart was suspended in a pericardial cradle. Micromanometer tipped catheters (Millar Instruments) were inserted into the right atrium through the external jugular vein and into the left ventricular cavity through the left ventricular apex. A 7 French balloon tipped catheter was placed in the coronary sinus. The proximal portions of the left anterior descending and left circumflex coronary arteries were dissected free from surrounding tissue. Ultrasonic flow probes (series SC, Transonics) were placed on both arteries and connected to a digital flow meter (model T206, Transonics) to monitor epicardial CBF. A 20 gauge polyethylene catheter was inserted into the distal portion of the left anterior descending coronary artery in the group 1 dogs.

\section{Haemodynamic measurements}

All catheters were interfaced with a multichannel recorder (model ES 2000, Gould). Mean CBF and pressures were acquired digitally into a computer and the signals were displayed on-line using Labtech Notebook (Laboratory Technologies). CDP was calculated as the difference in the mean distal coronary and right atrial pressures in the group 1 dogs, and between the mean aortic and right atrial pressures in the group 2 dogs. Coronary vascular resistance (CVR) was calculated by dividing CDP by the CBF.

\section{Myocardial oxygen consumption and demand determinations}

Blood $(0.8 \mathrm{ml})$ was collected simultaneously from the femoral artery and coronary sinus in a $1 \mathrm{ml}$ heparinised syringe. Samples were immediately placed on ice and analysed within 60 minutes using a blood gas analyser (model 288, Ciba-Corning). From each sample, the partial pressure of oxygen, oxygen saturation, and haemoglobin were measured, from which the oxygen content was calculated. Myocardial oxygen consumption $\left(\mathrm{MV}_{\mathrm{O}_{2}}\right)$ was calculated by multiplying CBF by the difference in arterial and venous oxygen content. Myocardial oxygen demand was calculated by multiplying heart rate by the end systolic wall stress. End systolic wall stress was derived using the equation: [aortic systolic pressure $\times$ left ventricular cavity radius $] /[2 \times$ end systolic wall thickness $]$.

\section{MCE derived MBV and MBF}

A Sonos-5500 system (Phillips Ultrasound) was used for ultrasound imaging. Ultrasound was transmitted at 2.1 $\mathrm{MHz}$

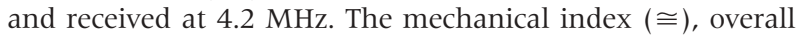
gain, compression (maximum), depth $(8 \mathrm{~cm})$, and focus $(8 \mathrm{~cm})$ were optimised at the beginning of each experiment and were held constant during the entire protocol. All backscatter measurements were done using acoustic densitometry, which involves measurements obtained before log compression and after processing of the ultrasound signal; they thus provide a linear relation between ultrasound signal and microbubble concentration over a wider range than do video intensity measurements obtained from conventional $\mathrm{B}$ mode images recorded on videotape. Images were obtained using intermittent harmonic imaging during a continuous infusion of BR-14, a novel ultrasound contrast agent comprised of microbubbles with perfluorocarbon gas contained within a lipid/surfactant shell. ${ }^{16}$ After steady state was achieved, MCE was undertaken with ultrasound gated to the ECG at end systole. Images were acquired at pulsing intervals of one, two, three, five, eight, 10, and 20 cardiac cycles to allow for progressively greater bubble replenishment of the ultrasound beam elevation. ${ }^{15}$ At least five images were obtained at baseline (precontrast) and at each pulsing interval before being transferred to optical disk.

Regions of interest were placed over the myocardium in images acquired at baseline (background) and during all pulsing intervals, as well as over the left ventricular cavity at baseline (background) and at a pulsing interval of 20 cardiac cycles. Pulsing interval versus background subtracted acoustic density plots were generated off-line and fitted to an exponential function, $y=A\left(1-e^{-\beta t}\right)$, where $y=$ acoustic density at pulsing interval $t, A=$ acoustic density after the ultrasound beam is completely replenished, and $\beta=$ rate constant that reflects the mean microbubble velocity. ${ }^{15} \mathrm{MBV}$ fraction was then calculated as the ratio between myocardial $A$ and the background subtracted acoustic density from the left ventricular cavity. ${ }^{15}$ The product, $A \cdot \beta$, was calculated to derive myocardial blood flow (MBF). Acoustic density measurements were also taken in the left ventricular cavity at end diastole and end systole in group 2 dogs during phenylephrine infusion, to determine whether the changes in left ventricular pressure affected microbubble size and therefore backscatter. These measurements were also made at a pulsing interval of 20 cardiac cycles.

\section{Wall thickness and thickening measurements}

Echocardiography was used to measure end diastolic and end systolic wall thickness and the left ventricular cavity diameter from two consecutive cardiac cycles. Wall thickness was measured in four quadrants which were kept constant throughout all stages within each dog. These measurements were then averaged. The left ventricular cavity diameter was measured at the same site in all stages. Percent wall thickening was calculated by dividing the difference between end systolic and end diastolic thickness by end diastolic thickness.

\section{Experimental protocol}

MCE and haemodynamic data, as well as arterial and coronary sinus blood samples, were collected at baseline and at each stage. In group 1 dogs, CDP was reduced by 10 $80 \mathrm{~mm} \mathrm{Hg}$ below baseline measurements by tightening the custom designed screw occluder placed on the coronary artery. In group 2 dogs, CDP was increased by raising the aortic pressure by $20-80 \mathrm{~mm} \mathrm{Hg}$ above baseline with a phenylephrine infusion (10-30 mg mixed in l litre of Plasmalyte).

\section{Statistical methods}

Comparisons between stages were undertaken using repeated measures analysis of variance (ANOVA). When a difference was found, a paired Student's $t$ test was used for interstage comparisons. Correlations were sought using least squares fitted linear regression analysis. Non-linear data were fitted using appropriate exponential or polynomial functions. A probability value of $\mathrm{p}<0.05$ (two sided) was considered statistically for all comparisons.

\section{RESULTS}

\section{Group 1 dogs}

The mean aortic pressure, left ventricular $\mathrm{dP} / \mathrm{dt}$, and myocardial oxygen demand did not change significantly with increasing stenosis levels in the group 1 dogs. Figure 1 shows selected haemodynamic and MCE data from these dogs, where the stenosis severity was judged by the magnitude of CDP decline. As coronary driving pressure fell, both epicardial 

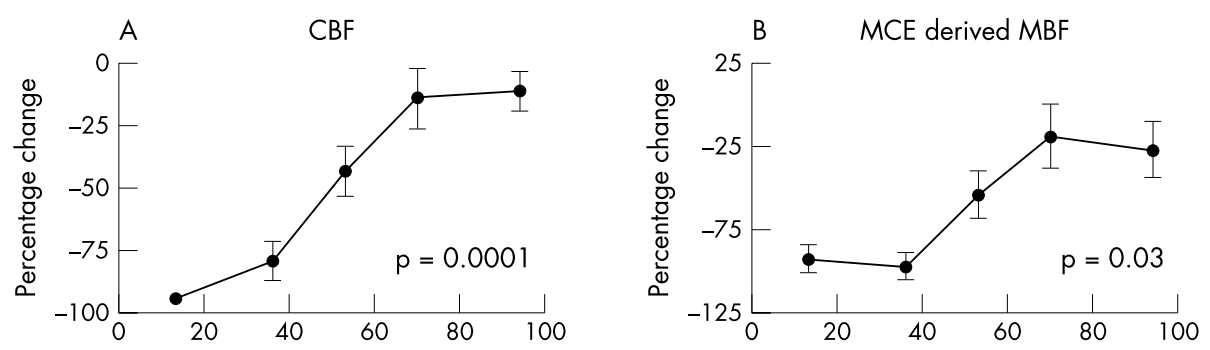

Figure 1 Relation between coronary driving pressure ( $x$ axis) and percentage change ( $y$ axis) of

(A) coronary blood flow (CBF), (B) myocardial contrast derived myocardial blood flow (MCE derived $\mathrm{MBF}),(\mathrm{C})$ myocardial blood volume (MBV) fraction, and (D) total myocardial vascular resistance when coronary driving pressure was decreased below the lower limit of autoregulation. The percentage change of each variable is compared to

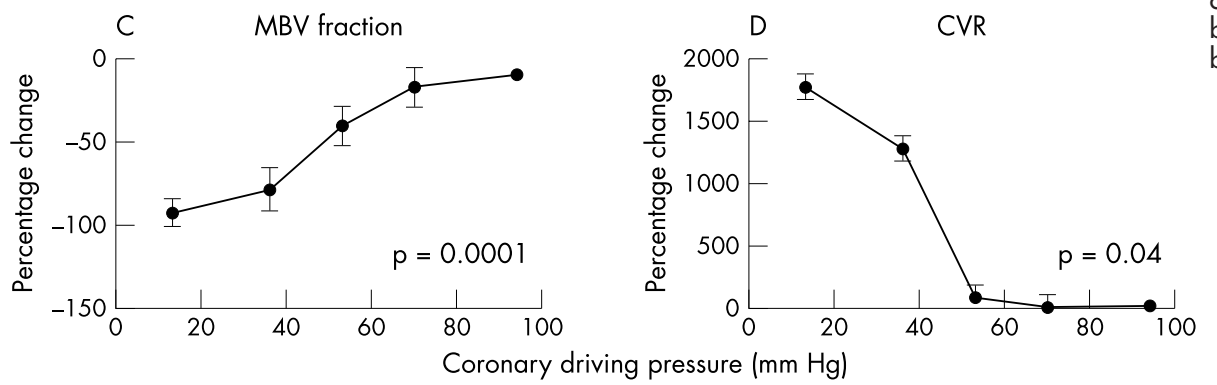
baseline values. Data are means, error bars $=$ SEM.

CBF and MCE derived MBF decreased (panels A and B). MBV fraction decreased with a reduction in CDP (panel C), which implies that capillary resistance increased (as capillaries cannot change in length). Consequently, total CVR increased (panel D). The decrease in MCE derived MBF was caused not only by a decrease in MBV fraction but also by a decrease in the mean microbubble velocity $(\mathrm{p}=0.04)$.

End diastolic mean wall thickness and left ventricular cavity size remained unchanged over the wide range of CDPs. However, mean wall thickness decreased $(p=0.002)$ and left ventricular cavity size increased $(p=0.006)$ with lower CDPs at end systole. As expected, per cent wall thickening also decreased significantly $(\mathrm{p}=0.001)$ with lower CDPs, owing to the pronounced wall thickening abnormality seen in the region supplied by stenosis. As percent wall thickening decreased, there was a trend for MCE derived MBF to decrease as well, although this did not reach statistical significance (fig 2).

\section{Group 2 dogs}

As expected, an increase in mean aortic pressure resulted in an increase in CDP and left ventricular $\mathrm{dP} / \mathrm{dt}$ with increasing doses of phenylephrine. Selected haemodynamic and MCE data from the group 2 dogs are shown in fig 3. When CDP was increased above the autoregulatory range, as expected $\mathrm{CBF}$ also increased (panel A); MCE derived MBF, however, remained unchanged (panel $\mathrm{B}$ ). The main reason for the lack of increase in MCE derived MBF was a decrease in MBV fraction (panel $\mathrm{C}$ ). Although mean microbubble velocity increased $(p=0.03)$ with increasing CDP, it did not offset the decrease in MBV fraction. Unlike in group 1 dogs, where a decrease in MBV fraction resulted in an increase in CVR, in group 2 dogs the decrease in MBV fraction was associated with a decrease in CVR.

The mismatch between epicardial CBF and MCE derived $\mathrm{MBF}$, as well as the decrease in CVR in the group 2 dogs, implies the presence of coronary arteriovenous shunting, which was supported by a progressive increase in the coronary sinus $\mathrm{PO}_{2}$ with higher CDPs (fig 4A). Coronary sinus $\mathrm{PO}_{2}$ increased because the increase in myocardial oxygen demand was not associated with a proportionate increase in $\mathrm{MVO}_{2}$ (fig 4B).

Wall thickness decreased, though not significantly, and left ventricular cavity size increased $(p=0.03)$ at end diastole with higher CDPs. Although systolic wall thickness tended to be smaller at higher CDPs at end systole, this also did not reach significance $(p=0.06)$. Left ventricular cavity size increased $(p=0.006)$ at end systole with increasing CDPs. There was a significant correlation between increasing percent wall thickening and increasing CDPs (fig 2). The relation between changes in MCE derived MBF and percent wall thickening was not significant. For the same incremental change in percent wall thickening, there was a greater percent change in CDP than in MCE derived MBF.

The backscatter from the left ventricular cavity was similar between end diastole and end systole during phenylephrine infusion (mean (SEM): 499 (399) v 492 (389)), indicating that the microbubble size did not change appreciably with left ventricular cavity pressure.

\section{$M B V$ over the entire range of CDP}

Figure 5 shows the effect of changes in CDP on CBF and MBV fraction over the entire range of CDPs. The data are derived from all 18 dogs. Within the autoregulatory range, changes in CDP did not result in significant changes in either CBF or MBV fraction. Below the autoregulatory range, both CBF and MBV fraction decreased. Above the autoregulatory range, while CBF increased, MBV fraction decreased.

\section{DISCUSSION}

\section{Relation between CDP and MBV}

It has been shown previously that when CDP is held within the range of 45-120 mm Hg, CBF remains unchanged. ${ }^{17}{ }^{18}$ It has also been shown that as CDP is reduced from normal levels down to $45 \mathrm{~mm} \mathrm{Hg}$, the total coronary blood volume (blood residing in the entire coronary tree) increases modestly because of arteriolar vasodilatation. ${ }^{18}{ }^{19}$ In this study, we show that MBV (the blood present only within the myocardium and mostly in capillaries) does not change in this range of CDP. This is consistent with the fact that when resting CDP is within the autoregulatory range, regulation of $\mathrm{CBF}$ is mediated solely through arterioles, which constitute only a small portion of the MBV and therefore would not be expected to change myocardial acoustic density.

In studies involving the skeletal muscle microcirculation, it has been found that capBV does not change within the autoregulatory range. Arterioles have been shown to be the principal site of resistance at these pressures. ${ }^{20}$ It is assumed that capHP stays fairly constant over a wide range of 


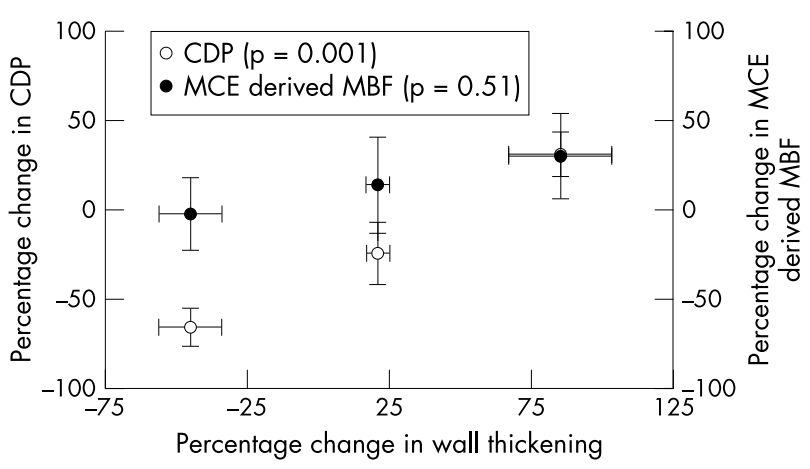

Figure 2 Relation between percentage change in wall thickening ( $x$ axis) and percentage changes in coronary driving pressure (CDP) and MBF over the entire range of CDPs.

perfusion pressures as the net transcapillary fluid movement has been found to remain relatively stable both within the autoregulatory range and beyond its lower and upper limits. ${ }^{1}$ Thus the findings from our study in the canine heart are consistent with previous reports using skeletal muscle preparations, which showed that capBV does not change appreciably within the autoregulatory range.

When CDP is decreased below $45-50 \mathrm{~mm} \mathrm{Hg}$, as expected $\mathrm{CBF}$ also decreases. ${ }^{18}$ We have previously shown that in this situation total coronary blood volume decreases as well. ${ }^{18}$ Results from our previous ${ }^{18}$ and current study indicate that in this setting CVR increases in proportion to a decrease in MBV. Consequently, when CBF starts decreasing, arterioles are maximally vasodilated, so any decrease in total coronary blood volume has to occur in some other vascular compartment (either capillaries or venules). In this study we have shown that the decrease in coronary blood volume reflects a decrease in MBV (or capBV), which results in an increase in CVR despite maximum dilatation of both coronary arterioles and venules.

When CDP is increased above $120 \mathrm{~mm} \mathrm{Hg}$, the resistance offered by arterioles is maximal and their ability to constrict is exhausted. As expected, therefore, CBF is influenced solely by the CDP. ${ }^{1}$ As CDP and CBF increase, microbubble velocity also increases. However, MBV decreases, resulting in no change in MCE derived MBF despite a significant increase in
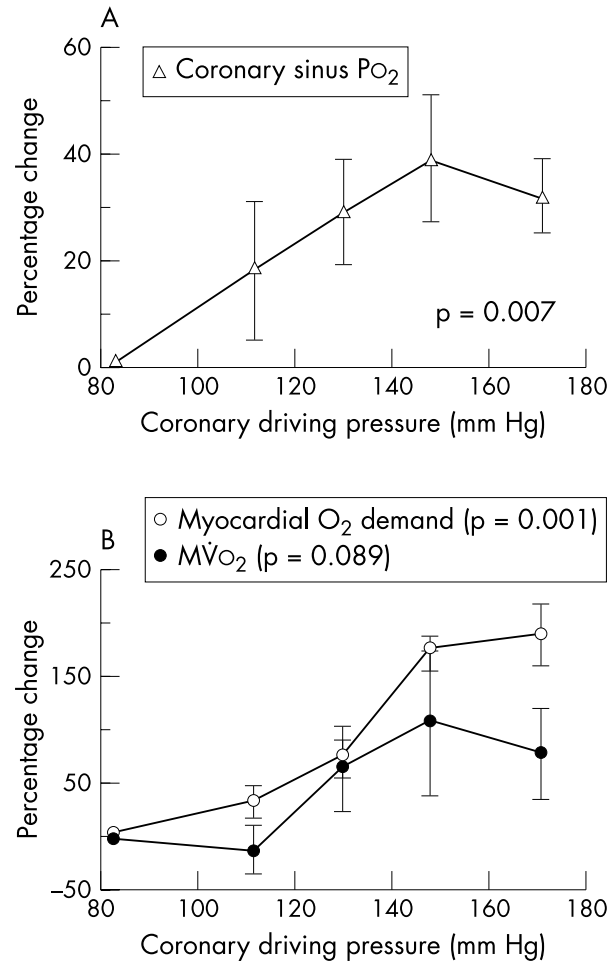

Figure 4 (A) Relation between coronary driving pressure ( $x$ axis) and changes in coronary sinus oxygen pressure $\left(\mathrm{PO}_{2}\right)$ (y axis) during phenylephrine infusion. (B) Relation between coronary driving pressure ( $x$ axis) and myocardial oxygen demand and myocardial oxygen consumption $\left(\mathrm{MVO}_{2}\right.$ ) (y axis) during phenylephrine infusion. Data are means, error bars $=$ SEM.

CBF. This occurs because of a decrease in MBV. Of interest, although $\mathrm{CBF}$ increases in proportion to increased myocardial oxygen demand caused by increasing $\mathrm{CDP}, \mathrm{MVO}_{2}$ does not increase proportionately. Furthermore, CVR decreases despite both a decrease in MBV and maximal resistance offered by precapillary arterioles. Thus it appears that the decrease in MBV is associated with shunting of excess CBF to the coronary venous system at sites that may lie proximal to the resistance vessels.
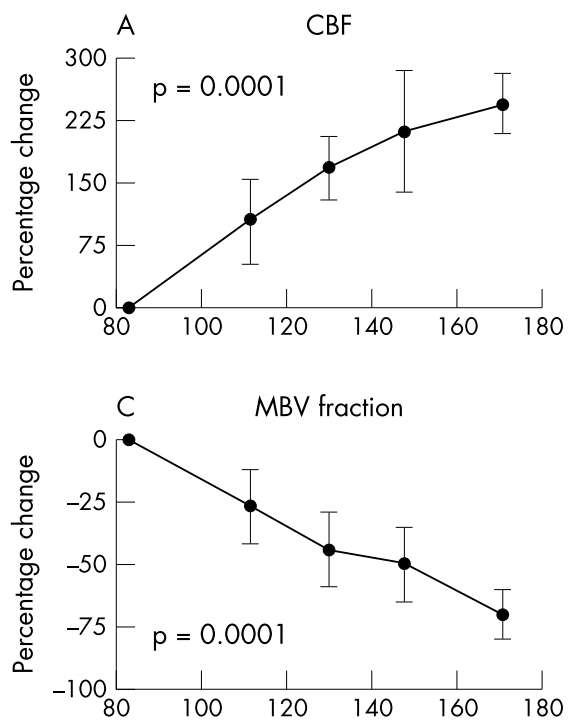
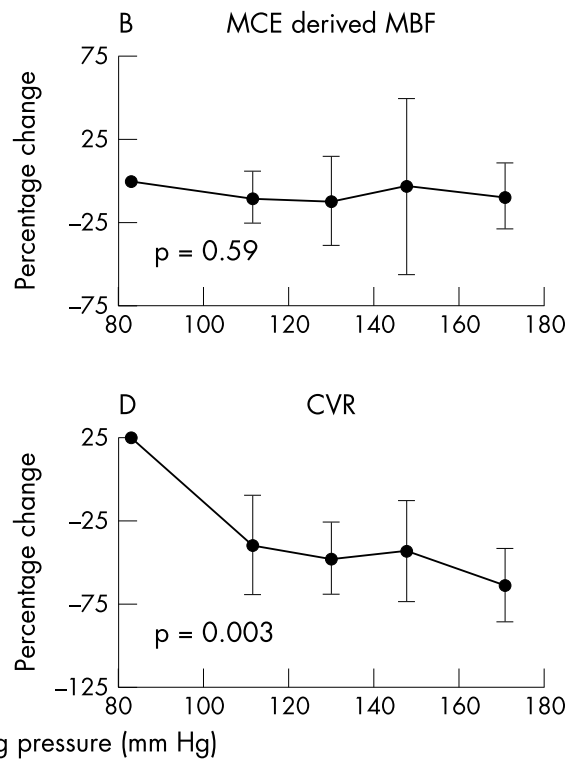

Figure 3 Relation between coronary driving pressure ( $x$ axis) and percentage change ( $y$ axis) of (A) coronary blood flow (CBF) (B) myocardial contrast derived myocardial blood flow (MCE derived $\mathrm{MBF}),(\mathrm{C})$ myocardial blood volume (MBV) fraction, and (D) total myocardial vascular resistance when coronary driving pressure was increased above the upper limit of autoregulation. The percentage change of each variable is compared to baseline values. Data are means, error bars $=$ SEM 


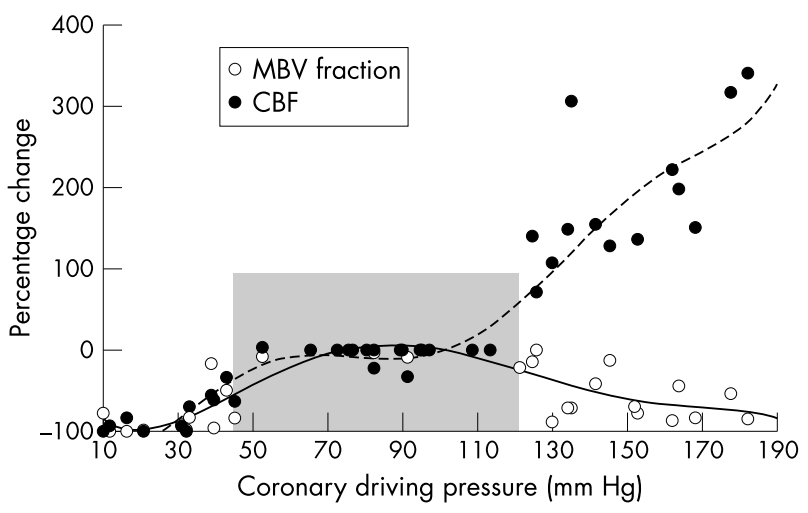

Figure 5 Relation between coronary driving pressure ( $x$ axis) and normalised percentage change in coronary blood flow (CBF) and myocardial blood volume (MBV) fraction (y axis) over a wide range of coronary driving pressures in all 18 dogs. The shaded area denotes the autoregulatory range. Data are means, error bars $=$ SEM.

Arteriovenous shunts have been shown to exist in the myocardium but are believed to be non-functional under physiological conditions. ${ }^{21}$ In a canine experiment, increasing CDP to $170 \mathrm{~mm} \mathrm{Hg}$ in the presence of hypoxia resulted in a nearly $50 \%$ increase in shunt flow. ${ }^{22}$ Another study in a similar model reported an increase in coronary arteriovenous shunting from $4.2 \%$ to $8.7 \%$ when CDP was increased from 97 to $177 \mathrm{~mm} \mathrm{Hg}$, with an associated decrease in arterialcoronary sinus $\mathrm{PO}_{2}$ difference. ${ }^{23}$ In our current study we also found that coronary sinus $\mathrm{PO}_{2}$ content increased with increasing $\mathrm{CDP}$, supporting the existence of functional arteriolar-venular shunts at raised CDPs. Because MBV decreased at these high CDPs, our results indicate that these shunts are proximal to the capillary bed.

We have developed a mathematical model to explain the above results (see the appendix and fig 6). Within the autoregulatory range, resistances from arterioles, capillaries, and venules-which are arranged in series-participate in the local regulation of MBF. Our model predicts that at a CDP below the autoregulatory range, an increase in capillary resistance alone is sufficient to maintain a constant capHP. However, when the CDP exceeds the autoregulatory range, arteriolar-venous shunts, arranged in parallel with the capillary bed, regulate local MBF in concert with capillaries. Our model predicts that shunt resistance decreases in order to accommodate the excess $\mathrm{CBF}$, which accounts for the noted decrease in CVR despite a decrease in MBV. As a result, these shunts-which are non-functional when CDP is within the autoregulatory range-are recruited when CDP exceeds the upper limit of autoregulation. The precise mechanisms by which capillaries and arteriolar-venous shunts maintain local MBF regulation remain to be elucidated.

\section{capHP $\mathbf{v}$ myocardial $\mathrm{O}_{2}$ delivery}

In this study we hypothesised that in an attempt to maintain capHP, capillaries will change their volume even at the risk of not meeting myocardial oxygen demand. This is most strikingly exemplified when CDP is increased above $120 \mathrm{~mm} \mathrm{Hg}$ and the arterioles are maximally constricted. In this situation if capBV remains unchanged, capHP would increase obligatorily. In an attempt to circumvent such an occurrence, MBV decreases, thus increasing capillary resistance. This decrease in $\mathrm{MBV}$ causes a decrease in $\mathrm{MV}_{2}$ despite a significant increase in myocardial oxygen demand. It appears, therefore, that capillary homeostasis takes precedence over myocardial oxygen delivery.
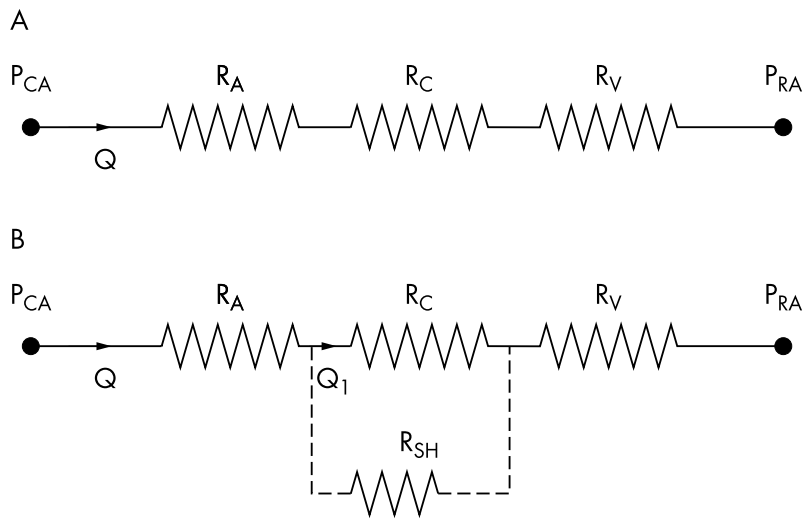

Figure 6 Mathematical models used to explain our findings.

(A) Graphic representation of the relation between epicardial coronary blood flow $(Q)$ and resistances between capillary pressure $\left(P_{C A}\right)$ and right atrial pressure $\left(P_{R A}\right)$ within the autoregulatory range. $(B)$ Graphic representation of the relation between $Q$ and resistances between $P_{C A}$ and $P_{R A}$ when coronary driving pressure exceeds the upper limit of the autoregulatory range. The capillary blood flow $\left(Q_{1}\right)$, as measured using myocardial contrast echocardiography, remains unchanged, either because of an increase in capillary resistance or because of a decrease in shunt resistance. $R_{A}=$ arteriolar resistance; $R_{C}=$ capillary resistance; $R_{S H}$, shunt resistance; $R_{V}=$ venular resistance.

When CDP decreases below $45 \mathrm{~mm} \mathrm{Hg}$, arteriolar vasodilatation is maximal and capHP becomes vulnerable to changes in CDP. If MBV remains constant, capHP will decrease with reduction in CDP. In order to prevent this situation from occurring, MBV decreases and capillary resistance increases. Consequently $\mathrm{MBF}$ may decrease out of proportion to the decrease in CDP, thus sacrificing myocardial oxygen delivery. Therefore it seems that even in this setting maintenance of capHP is more important than oxygen delivery.

In addition, below the autoregulatory range, percent wall thickening decreased as CDP and CBF decreased. This relation implies that the consequence of capillaries making an effort to maintain hydrostatic pressure is not only a reduction in myocardial oxygen delivery but also extends to a reduction in myocardial function. The capillaries and the adjacent myocytes and connective tissue can remain viable but are no longer functional.

\section{Critique of our methods}

Ultrasound signals are related to the 6th power of the microbubble radius. ${ }^{24} \mathrm{~A}$ small change in microbubble size can therefore result in a major change in signal. Being compressible, microbubbles are affected by the ambient pressure and have been shown to decrease in size at higher pressures. ${ }^{25} 26$ Thus microbubble size can decrease at the high systolic pressures induced by phenylephrine. However, we found that there was no difference in microbubble backscatter from the left ventricular cavity between end diastole and end systole. Moreover, as microbubbles within the myocardium and within the left ventricular cavity were subjected to the same high pressures, we were able to minimise any potential effect of pressure on ultrasound signal by normalising the myocardial signal to that in the left ventricular cavity.

Ultrasound signal can also be affected by changes within the myocardium, such as oedema, irrespective of whether microbubbles have been injected or not. It is for this reason that measurements were made from background subtracted images. By displacing capillaries connected to myocytes, myocardial oedema caused from increased capHP 
could also result in an apparent decrease in MBV. We therefore made measurements of left ventricular wall thickness and percent wall thickening and were unable to show any increase in thickness. Similarly, ischaemia resulting from lower CDPs can also result in oedema and affect myocardial acoustic intensity measurements. If this had occurred in our model, we would have expected to see an increase in wall thickness at the lower CDPs. Instead our wall thickness measurements did not change at end diastole and they decreased at end systole. Thus we are confident that myocardial oedema did not contribute to the reduction in MBV in either group where CDP was severely increased and decreased.

MBV can also increase from increased myocardial oxygen demand because of capillary recruitment..$^{27}{ }^{28}$ Thus one would have expected MBV to increase with an increase in afterload. Instead, the decrease in MBV reiterates the importance of maintaining a constant capHP even at the expense of myocardial oxygen delivery.

We assume that the constant net filtration across the capillary bed is the result of a constant capHP. In fact, for net filtration to be constant, the gradient between capillary and interstitial pressure must also be constant. During cardiac contraction the elastic impedance of the myocardium is continuously changing. When the myocardial pressure rises in systole, the squeezing of arterioles results in retrograde systolic flow in the larger arterioles and intramyocardial arteries, while the squeezing of the veins results in forward systolic flow into the coronary sinus. The increased resistance in the arterioles and veins functionally isolates the capillaries from the rest of the coronary circulation in systole. The capillaries face an increased myocardial pressure but do not change their volume, as the capHP (now a closed chamber within the myocardium) also rises with the myocardial pressure. ${ }^{29}$ Thus the increase in interstitial pressure during systole does not result in reduced net filtration. It is unlikely, therefore, that further change in elastic impedance induced by phenylephrine will affect capBV. We have also extrapolated findings from skeletal muscle to myocardium. However, this concept of constant hydrostatic pressure is so fundamental that it is unlikely to differ between different types of muscle.

We intentionally did not use radiolabelled microspheres for assessment of myocardial perfusion as they provide us with total flow to myocardial arterioles up to $13 \mu \mathrm{m}$ in size. The assumption is that whatever will reach these arterioles will also reach capillaries. While this is true under normal circumstances, it may not be true when aortic pressure is increased and arteriovenous shunts become operative. It is not known at what level of the circulation such shunts are present. We have previously shown that when flow to the myocardium is delivered retrogradely through the coronary sinus, Thebesian veins are present even between $13 \mu \mathrm{m}$ venules and capillaries, so that flow to the capillaries is substantially less than to the small venules. ${ }^{30}$ It is for this reason that we used MCE, which has been well validated for assessing capillary flow not only in the myocardium but in other organs as well.

Our observations suggest that maintaining constant capHP and homeostasis takes precedence over oxygen delivery. However, we were not able to document an increase in coronary sinus lactate concentration. One explanation is that in group 1 dogs, the stenosis was created only in one myocardial bed, which may not have affected the mean lactate concentration in the coronary sinus, which receives blood from many myocardial regions. This situation, however, does not apply when CDP is increased, as the entire left ventricle is exposed to the same raised pressures. The lack of any change in lactate extraction may therefore be related to the failure of this variable to reflect mild to moderate ischaemia. $^{31} 32$

Finally, the design of our study and the methods used do not permit an evaluation of the proximate mechanism of the decrease in MBV. As the changes occur immediately, it has been suggested that they are modulated by myogenic reflexes, particularly in skeletal muscle. ${ }^{9}$ It has also been suggested that, at least in skeletal muscle, viscosity changes in small venules may be responsible for maintaining a constant capHP. ${ }^{33}$ More intriguing are the putative signals that may initiate changes in MBV, and the source of these signals.

\section{Clinical implications}

We have demonstrated that capillaries participate in the control of resting MBF when active arteriolar vasomotion (autoregulation) is no longer functioning. When CDP is between $45-120 \mathrm{~mm} \mathrm{Hg}$ and arteriolar vasomotion is operative, $\mathrm{CBF}$ is maintained by changes in arteriolar resistance. In this setting, capillary resistance and MBV remain constant. When arteriolar tone is minimal at CDP below $45 \mathrm{~mm} \mathrm{Hg}$, capillaries participate in the regulation of MBF. When arteriolar tone is maximal at CDP above $120 \mathrm{~mm} \mathrm{Hg}$, both capillaries and arteriolar-venular shunts participate in the regulation of MBF. In both instances, MBV decreases in order to maintain a constant capHP. The regulation of CHP takes precedence over myocardial oxygen delivery.

The clinical implications of these novel findings can be related to periods of acute rise or fall in aortic pressure. In the former, capillary derecruitment allows maintenance of capHP which prevents myocardial oedema and even haemorrhage; in the latter, capillary derecruitment also allows maintenance of capHP so that myocellular and interstitial volume are not redistributed to the blood pool, and homeostasis is not disturbed. Eventually, however, the increased capillary resistance can contribute to myocardial ischaemia and cell death. Therefore, bringing the aortic pressure to normal levels would obviously be the first measure so that autoregulation can be restored and capillary resistance returned to normal.

\section{ACKNOWLEDGEMENTS}

Supported in part by grants from the National Institutes of Health (3R01-HL48890) and Bracco Research SA, Geneva, Switzerland. Phillips Ultrasound (Andover, Massachusetts, USA) provided the ultrasound equipment. DEL was the recipient of a postdoctoral training grant (T32-HL07355) from the National Institutes of Health. KW and JRL were the recipients of the mentored clinical scientist development award (K08-HL03909 and K08-HL03810) from the National Institutes of Health. MPC was the recipient of a medical student research grant from the American Diabetes Association. The study was presented in part at the young investigator award competition of the 49th Annual Scientific Session of the American College of Cardiology in Atlanta, Georgia

\section{Authors' affiliations}

D E Le, A R Jayaweera, K Wei, M P Coggins, J R Lindner, S Kaul, The Cardiovascular Imaging Center, the Cardiovascular Division, University of Virginia School of Medicine, Charlottesville, Virginia, USA

\section{APPENDIX}

\section{MATHEMATICAL MODELLING OF OUR RESULTS}

Based on the coronary vasculature model we have used before $^{13}$ and shown in fig 6A, the mean capillary pressure $\left(P_{c a p}\right)$ measured at the centre of the capillary bed is given by the equation: 


$$
P_{\text {cap }}=\frac{\left(R_{a}+\frac{R_{c}}{2}\right) P_{R A}+\left(\frac{R_{c}}{2}+R_{v}\right) P_{C A}}{\left(R_{a}+R_{c}+R_{v}\right)}
$$

where $R_{a}, R_{c}$, and $R_{v}$ are arteriolar, capillary and venular resistances, respectively, and $P_{R A}$ and $P_{C A}$ are right atrial and coronary artery pressures. It is clear from this expression that changes in $P_{C A}$ will be associated with directionally similar changes in $P_{c a p}$. The direction in which $P_{c a p}$ will change with $R_{c}$ is not intuitively apparent from this equation. However, if we take the partial derivative of $P_{c a p}$ with respect to $R_{c}$ we obtain the following equation:

$$
\frac{\partial P_{c a p}}{\partial R_{c}}=\frac{\left(P_{C A}-P_{R A}\right)\left(R_{a}-R_{v}\right)}{2\left(R_{a}+R_{c}+R_{v}\right)^{2}}
$$

As $P_{C A}>P_{R A}$ and $R_{a}>R_{v}$, the partial differential is always positive, which means that $P_{c a p}$ increases with an increase in $R_{c}$.

Therefore, when $P_{C A}$ decreases below the lower limit of the autoregulatory range, $P_{c a p}$ can be maintained constant by an increase in $R_{c}$. When $P_{C A}$ increases beyond the upper limit of the autoregulatory range, an increase in $R_{c}$ alone cannot maintain a constant $P_{\text {cap }}$. This is the reason why we believe that arteriovenous shunts become operative at a high CDP and divert excess flow away from the capillaries.

We therefore propose a modified circuit depicted in fig 6B, where $R_{s h}$ is the shunt resistance and $Q$ and $Q_{1}$ are the epicardial and capillary flows, respectively. The equivalent resistance $R_{e q}$ of this parallel combination, $\left(R_{c} \cdot R_{s h}\right) /\left(R_{c}+R_{s h}\right)$, is always $\leqslant R_{c}$ and can be decreased by decreasing either or both of $R_{c}$ or $R_{s h}$. Under normal perfusion pressures, $R_{s h}>>R_{c}$ so that $R_{e q}=R_{c}$. However, when $P_{C A}$ rises above the autoregulatory limit, although $R_{c}$ increases, a sufficient decrease in $R_{s h}$ is also necessary to result in a decrease in $R_{e q}$ in order to maintain the same $P_{\text {cap }}$.

The reason why $R_{c}$ increases at high CDP may be explained by the experimental finding that while epicardial CBF $Q$ rises, the capillary flow $Q_{1}$ (measured using MCE) remains unchanged. $Q_{1}$ is related to $Q$ by the equation $Q_{1}=Q-R_{S h} /$ $\left(R_{c}+R_{s h}\right)=Q /\left[1+\left(R_{c} / R_{s h}\right)\right]$. According to this equation, when $Q$ increases, $Q_{l}$ can be maintained constant by either increasing $R_{c}$ and/or decreasing $R_{s h}$.

When the precapillary pressure rises because of an increase in CDP, $P_{\text {cap }}$ can be maintained by increasing the pressure gradient across the capillaries, which can be achieved by increasing either or both $Q_{1}$ and $R_{c}$. We observed that $Q_{1}$ remained unchanged and that capBV decreased, implying an increase in $R_{c}$. Accordingly, the increase in $R_{c}$ accounts for the increase in the capillary pressure gradient $\left(Q_{I} \cdot R_{c}\right)$, which in turn accounts for the increase in the capillary blood flow velocity $(\beta)$ that we observed on MCE. Above the autoregulatory range, $R_{a}$ and $R_{v}$ are maximal and constant. As $P_{C A}$ increases, $R_{e q}$ decreases and therefore the total myocardial resistance $\left(R_{a}+R_{e q}+R_{v}\right)$ decreases, which is in agreement with our experimental observations.

\section{REFERENCES}

1 Rouleau J, Boerboom LE, Surjadhana A, et al. The role of autoregulation and tissue diastolic pressures in the transmural distribution of left ventricular blood flow in anesthetized dogs. Circ Res 1979;45:804-15.
2 Chilian WM, Eastham CL, Marcus ML. Microvascular distribution of coronary vascular resistance in beating left ventricle. Am J Physiol 1986;251:H779-88.

3 Chilian WM, Layne SM. Coronary microvascular responses to reductions in perfusion pressure. Evidence for persistent arteriolar vasomotor tone during coronary hypoperfusion. Circ Res 1990;66:1227-38.

4 Habazettl $H$, Vollmar $M$, Christ $H$, et al. Heterogeneous microvascular coronary vasodilation by adenosine and nitroglycerin in dogs. J Appl Physiol 1994;76:1951-60.

5 Kanatsuka HK, Lamping G, Eastham CL, et al. Heterogenous changes in epimyocardial microvascular size during graded coronary stenosis. Evidence of the microvascular site for autoregulation. Circ Res 1990;66:389-96.

6 House SD, Johnson PC. Microvascular pressure in venules of skeletal muscle during arterial pressure reduction. Am J Physiol 1986;250:H838-45.

7 Kuo L, Arko F, Chilian WM, et al. Coronary venular responses to flow and pressure. Circ Res 1993;72:607-15.

8 Kassab GS, Lin DH, Fung YB. Morphometry of pig coronary venous system. Am J Physiol 1994;267:H2100-13.

9 Järhult J, Mellander S. Autoregulation of capillary hydrostatic pressure in skeletal muscle during regional arterial hypo- and hypertension. Acta Physiol Scand 1973;91:32-41.

10 Kaul S. Myocardial contrast echocardiography: 15 years of research and development. Circulation 1997;96:3745-60.

11 Kaul S, Jayaweera AR. Coronary and myocardial blood volumes: noninvasive tools to assess the coronary microcirculation? Circulation 1997;96:719-24.

12 Dawson D, Vincent MA, Clark A, et al. Vascular recruitment in skeletal muscle during exercise. Am J Physiol 2002;282:E714-20.

13 Bosman J, Tangelder GJ, Oude Egbrink MGA, et al. Capillary diameter changes during low perfusion pressure and reactive hyperemia in rabbit skeletal muscle. Am J Physiol 1995;269:H1048-55.

14 Jayaweera AR, Wei K, Coggins M, et al. Role of capillaries in determining coronary blood flow reserve: New insights using myocardial contrast echocardiography. Am J Physiol 1999;277:H2363-72.

15 Wei K, Jayaweera AR, Firoozan S, et al. Quantification of myocardial blood flow with ultrasound-induced destruction of microbubbles administered as a constant venous infusion. Circulation 1998;97:473-83.

16 Schneider MA, Broillet A, Bussat $P$, et al. Gray-scale liver enhancement in VX2 tumor-bearing rabbits using BR14, a new ultrasonographic contrast agent. Invest Radiol 1997;32:410-17.

17 Gould KL, Lipscomb K. Effects of coronary stenoses on coronary flow reserve and resistance. Am J Cardiol 1974;34:48-55.

18 Lindner JR, Skyba DM, Goodman NC, et al. Changes in myocardial blood volume with graded coronary stenosis: an experimental evaluation using
myocardial contrast echocardiography. Am J Physiol 1997;272:H567-75.

19 Canty JM, Judd RM, Brody AS, et al. First-pass entry of nonionic contrast agent into the myocardial extravascular space. Effects on radiographic estimates of transit time and blood volume. Circulation 1991;84:2071-8.

20 Jones CJ, Kuo L, Davis MJ, et al. Distribution and control of coronary microvascular resistance. Adv Exp Med Biol 1993;346:181-8.

21 Wearn JT, Mettier SR, Klump TG, et al. The nature of the vascular communications between the coronary arteries and the chambers of the heart. Am Heart J 1933;9:143-5.

22 Downey FH, Bashour FA, Jishi B, et al. Arteriovenous shunts in dilated or reperfused canine coronary vasculature. Microvasc Res 1979;17:22-6.

23 Crystal GJ, Boatwright RB, Downey HF, et al. Shunting of microspheres across the canine coronary circulation. Am J'Physiol 1979;236:H7-12.

24 Albers VM. Underwater acoustic handbook. State College: The Pennsylvania State University Press, 1960.

25 Vandenberg B, Melton HE. Acoustic lability of albumin microspheres. J Am Soc Echocardiogr 1994; 7:582-9.

26 Vuille C, Nidorf M, Morrissey RL, et al. Effect of static pressure on the disappearance rate of specific echocardiographic contrast agents. J Am Soc Echocardiogr 1994;7:347-54.

27 Friedman BJ, Grinberg OY, Isaacs KA, et al. Myocardial oxygen tension and relative capillary density in isolated perfused rat hearts. J Mol Cell Cardiol 1995;27:2551-8.

28 Henquell L, Honig CR. Intercapillary distances and capillary reserve in right and left ventricles: significance for control of tissue $\mathrm{pO}_{2}$. Microvasc Res $1976 ; 12: 35-41$.

29 Wei K, Le DE, Bin JP, et al. Non-invasive detection of coronary artery stenosis at rest without recourse to exercise or pharmacologic stress. Circulation 2002;105:218-23.

30 Villanueva FS, Spotnitz WD, Glasheen WP, et al. New insights into the physiology of retrograde cardioplegia delivery. Am J Physiol 1995;268:H1555-66.

31 Apstein CS, Gravino F, Hood WB. Limitations of lactate production as an index of myocardial ischemia. Circulation 1979;4:877-87.

32 Sidi A, Davis RF. Lactate extraction fails to accurately reflect regional lactate production in ischemic myocardium. J Cardiothorac Anesth 1989;3:321-8.

33 Mellander S. On the control of capillary fluid transfer to pre-capillary and post-capillary vascular adjustments. A brief review with special emphasis on myogenic mechanisms. Microvasc Res 1978;15:319-30. 